\title{
Avaliação das queixas estético-funcionais em pacientes portadores de deformidades dentofaciais
}

\author{
Dario Ravazzi Ambrizzi*, Sergio Altino Franzi ${ }^{\star \star}$, Valfrido Antonio Pereira Filho***, \\ Marisa Aparecida Cabrini Gabrielli***, Carla Maria Melleiro Gimenez ${ }^{\star \star \star \star}$, Francisco A. Bertoz $z^{\star \star \star \star *}$
}

\begin{abstract}
Resumo
Objetivo: avaliar as queixas relatadas por pacientes portadores de deformidades dentofaciais como fatores motivadores na procura do tratamento ortodôntico-cirúrgico. Metodologia: foram verificados 130 prontuários de pacientes em tratamento no CEDEFACE - Araraquara, apresentando um período pós-operatório mínimo de 6 meses e idade média de 30,9 anos. A classificação dos tipos de deformidades foi realizada por meio de análise das telerradiografias e das fotografias, sendo subdivididas em três grupos, de acordo com suas características de perfil facial. As informações referentes às queixas foram colhidas por meio de questionários e divididas em duas categorias: queixa principal e queixas secundárias. Em relação à análise estatística, utilizaram-se medidas de tendência central (média e mediana) e de variabilidade (variação e desvio-padrão) para descrever as variáveis numéricas de idade. As variáveis categóricas de gênero, deformidades dentofaciais e queixas foram descritas através da distribuição de freqüências, com nível de significância de 5\%. Resultados: observou-se predominância do gênero feminino, na razão de 2:1. Na maioria das vezes, a deformidade requeria tratamento somente na maxila ou somente na mandíbula. No gênero feminino predominaram as deformidades de perfil convexo (46\%); no gênero masculino houve predominância de deformidades de perfil côncavo $(48,8 \%)$. As queixas principais evidenciaram que o anseio estético é predominante, tanto no gênero feminino quanto no masculino. Conclusão: os anseios de melhora estética foram predominantes em ambos os gêneros. A maioria dos pacientes também apresentou anseio por melhora funcional. Queixas de dor orofacial foram observadas em metade da casuística, sendo a segunda queixa mais relatada.
\end{abstract}

Palavras-chave: Deformidade dentofacial. Cirurgia ortognática. Diagnóstico. Tratamento. Queixas.

\footnotetext{
* Cirurgião bucomaxilofacial, responsável pelo Serviço de Cirurgia e Traumatologia Bucomaxilofacial dos Hospitais Escola Padre Albino e Emílio Carlos - Catanduva (SP). Mestre pelo Curso de Pós-Graduação em Ciências da Saúde do Hospital Heliópolis - HOSPHEL.

** Professor do Curso de Pós-Graduação em Ciências da Saúde do Hospital Heliópolis - HOSPHEL. Doutor em Medicina pela Faculdade de Medicina da Universidade de São Paulo. Cancerologista de Cabeça e Pescoço.

*** Professor Assistente Doutor da Disciplina de Cirurgia e Traumatologia Buco-Maxilo-Facial da Faculdade de Odontologia de Araraquara UNESP.

**** Mestre e Doutoranda em Ortodontia pela Faculdade de Odontologia de Araçatuba-Unesp. Ortodontista do CEDEFACE.

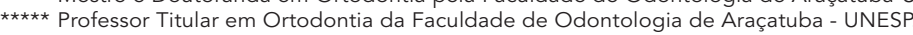




\section{INTRODUÇÃO}

Nas últimas décadas, o aperfeiçoamento técnico-científico ligado às áreas de Ortodontia e Cirurgia Ortognática possibilitou formas de planejamento integrado, com maior previsibilidade de resultados no tratamento de pacientes portadores de deformidades dentofaciais ${ }^{4}$.

Somadas ao fator estético inerente à deformidade facial, condições funcionais podem estar prejudicadas, como a mastigação, a fonação e a respiração nasal ${ }^{11}$. Problemas psicológicos relacionados à auto-estima e à sociabilidade também apresentam íntima relação com os conceitos de auto-imagem do paciente portador da deformidade. Vários autores também atribuem ou associam muitos relatos de dores orofaciais às desproporções do esqueleto mastigatório ${ }^{13,14,15}$.

O tratamento ortodôntico associado à cirurgia ortognática tem trazido soluções a esses pacientes, sendo relatado: melhora estética facial ${ }^{3,21}$, melhora das funções mastigatórias ${ }^{26}$, melhora das funções fonéticas ${ }^{12,23}$ e redução de dores articulares e miofaciais ${ }^{15,19}$. Apesar de as dificuldades funcionais estarem sempre presentes, são as queixas estéticas que, na maioria das vezes, impulsionam esses pacientes a procurar pelo tratamento ${ }^{7,24}$. A importância relativa da estética na sociedade atual é compreendida ao analisar-se os atributos positivos associados à atratividade física ${ }^{17}$. Em contrapartida, outros autores apresentam casuísticas onde as queixas funcionais são tão representativas quanto as estéticas ${ }^{5,20}$.

Analisando a literatura referente ao tratamento de deformidades dentofaciais, observa-se que maior ênfase tem sido dada aos aspectos técnicos do tratamento ortodôntico e da cirurgia ortognática e às opiniões subjetivas das equipes com relação aos resultados estético-funcionais obtidos, havendo menor atenção aos anseios e expectativas do paciente e à sua autoavaliação de acordo com os resultados após o tratamento ${ }^{25}$. O conhecimento das queixas estéticas e funcionais ocasionadas pela deformidade e dos aspectos psicossociais envolvi- dos amplia o contato direto entre profissionais e pacientes, fazendo-os chegar a um denominador comum, que será buscado, visando a satisfação do paciente com os resultados obtidos pelo tratamento ortodôntico e a cirurgia ortognática ${ }^{9,11}$.

Uma parcela significativa da população apresenta má oclusão dentária severa, a ponto de afetar as proporções faciais, devido às alterações de crescimento do esqueleto facial, notadamente na maxila e na mandíbula. Aproximadamente 10\% da população apresentam má oclusão Classe II e 2,5\% má oclusão Classe III, embora a necessidade de correção cirúrgica devido a uma deformidade dentofacial seja premente em aproximadamente $3 \%$ da população ${ }^{21}$.

Existe um predomínio do gênero feminino na procura pelo tratamento, com razões entre 2:1 e 3:1, também descrito pela literatura ${ }^{2,10,16}$. Proffit, Phillips e Dann ${ }^{18}$ afirmam que o gênero feminino se apresenta mais disposto a procurar e se submeter a tratamento ortodôntico e cirurgia ortognática, quando comparado ao gênero masculino.

O descontentamento com a estética facial é referido por muitos autores como o fator motivador mais freqüente na procura pela cirurgia ortognática ${ }^{8,17,24}$. Magnusson et al. ${ }^{14}$ encontraram anseios estéticos em 75\% de seus pacientes, embora quase sempre acompanhados de anseios de ordem funcional. Flanary, Barnwell e Alexander ${ }^{7}$ afirmam que a procura por estética é mais freqüente em pacientes do gênero feminino, mas, para Barbosa et al. ${ }^{1}$, os anseios estéticos são um importante fator de decisão pela cirurgia ortognática para ambos os gêneros, embora essas preocupações estéticas possam ser freqüentemente omitidas pelos pacientes, principalmente os do gênero masculino. Kiyak et al. ${ }^{10}$ observaram que os homens apresentavam mais motivos funcionais como razão primária para o tratamento, quando comparados às mulheres. Em compensação, um maior número de mulheres afirmou terem sido motivadas por desordens temporomandibulares e dores orofaciais, dados estes também relatados por 
outros autores ${ }^{17,20}$.

O paciente em tratamento ortodôntico précirúrgico está exposto a muitas informações a respeito de sua deformidade, transmitidas pela equipe profissional, por outros pacientes ou mesmo por materiais de ilustração e divulgação, podendo gerar, com isso, uma adição de motivações e expectativas ausentes no início do tratamento ${ }^{20}$.

Pelas razões expostas, o presente trabalho teve por objetivo determinar os motivos que levam os pacientes a procurarem por um tratamento ortodôntico-cirúrgico.

\section{MATERIAL E MÉTODOS}

Após a aprovação do projeto pelo Comitê de Ética em Pesquisa da Faculdade de Medicina de Catanduva/SP, 130 pacientes portadores de deformidades dentofaciais que se submeteram a tratamento ortodôntico-cirúrgico no CEDEFACE (Centro de Pesquisa e Tratamento das Deformidades Buco-Faciais de Araraquara), apresentando um período pós-operatório mínimo de 6 meses, foram avaliados. Foram excluídos pacientes fissurados, portadores de síndromes autossômicas, além de portadores de alterações psiquiátricas ou que acarretassem comprometimento das capacidades cognitivas.

A idade média dos pacientes foi de 30,9 anos (18 - 56 anos) e a mediana de 30 anos. Dos 130 pacientes avaliados, $87(66,9 \%)$ pertenciam ao gênero feminino, enquanto $43(33,1 \%)$ eram do masculino. Do toal, 117 pacientes (90\%) eram brancos, enquanto os não brancos totalizaram 13 pacientes (10\%).

A classificação dos tipos de deformidades foi realizada por meio de análise das telerradiografias (lateral e frontal), bem como pela análise das fotografias (perfil e frontal) contidas nos prontuários dos pacientes. As deformidades foram divididas e agrupadas em três grupos, de acordo com suas características:

- Grupo 1: deformidades cujas dimensões ântero-posteriores da maxila sobrepujam as da man- díbula, produzindo um perfil convexo (retrognatismo de mandíbula, prognatismo de maxila ou excesso vertical de maxila em paciente com má oclusão Classe II);

- Grupo 2: deformidades cujas dimensões ântero-posteriores da mandíbula sobrepujam as da maxila, produzindo um perfil côncavo (prognatismo mandibular, hipoplasia de maxila ou excesso vertical de maxila em paciente com má oclusão Classe III);

- Grupo 3: outras deformidades dentoesqueléticas onde não existe predomínio óbvio de dimensões ântero-posteriores entre as estruturas anatômicas (mordida aberta anterior com oclusão Classe I ou assimetria facial em norma frontal).

As informações referentes às queixas apresentadas como motivadoras para a procura por tratamento ortodôntico-cirúrgico foram colhidas, por meio de questionário realizado pelo autor, diretamente dos pacientes e subdivididas em duas categorias: queixa principal (fator primário motivador), como sendo o maior anseio do paciente; e queixas secundárias (outros fatores motivadores), embora em menor grau à queixa primária, podendo ser relatada mais de uma. As queixas se dividiram em: anseios estéticos, anseios funcionais (melhoras mastigatória, fonética e respiratória nasal), dor orofacial e pressão social.

Em relação à análise estatística, este estudo descritivo utilizou medidas de tendência central (média e mediana) e de variabilidade (variação e desvio-padrão) para descrever as variáveis numéricas de idade. As variáveis categóricas de gênero, deformidades dentofaciais e queixas foram descritas através da distribuição de freqüências. Para todos os testes, o nível de significância de 5\% foi adotado.

\section{RESULTADOS}

Dos 130 pacientes avaliados, 87 (66,9\%) pertenciam ao gênero feminino e $43(33,1 \%)$ ao masculino, resultando em uma razão de 2:1. Com relação à idade dos pacientes que procuraram tra- 
tamento, observamos a predominância da faixa etária de 20-39 anos, em ambos os gêneros.

No gênero feminino predominaram as deformidades do grupo 1 (46\%). Em contrapartida, houve predominância de deformidades do grupo 2 (48,8\%) no gênero masculino, mas ambos apresentaram distribuições significativas nas outras categorias. Esses resultados apresentaram significância estatística $(\mathrm{p}=0,015)$.

Em relação aos tipos de deformidades dentofaciais, os casos de deformidades tratadas por meio de uma única osteotomia foram predominantes $(57,7 \%)$, sendo observados, com maior freqüência, o retrognatismo mandibular e a hipoplasia de maxila, acometendo $42,3 \%$ e $36,1 \%$ da amostra, respectivamente. $\mathrm{O}$ acometimento de deformidades tratadas com osteotomias bima-

Tabela 1 - Incidência dos tipos de deformidades dentofaciais.

\begin{tabular}{ccc}
\hline deformidade dentofacial & $\mathbf{n}^{\circ}$ de pacientes & $\%$ \\
\hline retrognatismo mandibular & 55 & 42,3 \\
hipoplasia de maxila & 47 & 36,1 \\
mordida aberta anterior & 22 & 16,9 \\
excesso vertical de maxila & 20 & 15,4 \\
prognatismo mandibular & 18 & 13,8 \\
assimetria facial & 12 & 9,2 \\
prognatismo de maxila & 5 & 3,8 \\
\hline
\end{tabular}

Tabela 2 - Queixas primárias para a procura de tratamento ortodôntico-cirúrgico.

\begin{tabular}{ccc}
\hline queixa primária & $\mathbf{n}^{\circ}$ de pacientes & $\%$ \\
\hline estética & 76 & 58,5 \\
dor orofacial & 26 & 20 \\
dificuldade mastigatória & 22 & 16,9 \\
pressão social & 4 & 3,1 \\
dificuldade fonética & 1 & 0,8 \\
dificuldade de respiração nasal & 1 & 0,8 \\
\hline
\end{tabular}

Tabela 3 - Queixas secundárias para a procura de tratamento ortodôntico-cirúrgico.

\begin{tabular}{ccc}
\hline queixa secundária & $\mathbf{n}^{\circ}$ de pacientes & $\%$ \\
\hline dor orofacial & 36 & 27,7 \\
dificuldade mastigatória & 36 & 27,7 \\
estética & 23 & 17,7 \\
\cline { 2 - 3 } dificuldade fonética & 9 & 6,9 \\
dificuldade de respiração nasal & 5 & 3,8 \\
\cline { 2 - 3 } pressão social & 2 & 1,5 \\
\hline
\end{tabular}

xilares foi de 39\%. As incidências são detalhadas na tabela 1 .

As queixas primárias e secundárias apresentadas como fatores motivadores para a procura do tratamento ortodôntico-cirúrgico podem ser vistas nas tabelas 2 e 3 .

As queixas principais para a procura ao tratamento ortodôntico-cirúrgico evidenciaram que o anseio estético é predominante, tanto no gênero feminino quanto no masculino. Correspondendo, no feminino, a $60 \%$ das queixas principais, seguido pelas queixas de dor orofacial $(21,8 \%)$, dificuldade mastigatória $(13,8 \%)$, pressão social $(2,3 \%)$, dificuldade fonética $(1,1 \%)$ e dificuldade respiratória nasal $(1,1 \%)$. Em relação ao masculino, o anseio estético correspondeu a 55,8\% das queixas principais, seguido pelas queixas de dificuldade mastigatória $(23,2 \%)$, dor orofacial $(16,3 \%)$ e pressão social $(4,6 \%)$. Entretanto, estas diferenças observadas entre os gêneros não apresentaram significância estatística (Gráf. 1).

Em relação às queixas principais para a procura de tratamento, de acordo com o tipo de deformidade dentofacial, o anseio estético foi predominante nas três categorias, sendo responsável por $76,7 \%$ no grupo $2,57,1 \%$ no grupo 1 e por $42,8 \%$ da categoria outras deformidades. Queixas de dor orofacial foram relatadas por $26,5 \%$ do grupo 1 ; $22,8 \%$ do grupo 2 e 10,9\% do grupo 3. Queixas principais de dificuldade mastigatória também foram relatadas por $22,2 \%, 15,2 \%$ e $10,2 \%$, nos grupos 3, 2 e 1, respectivamente (Gráf. 2).

No questionamento sobre outras possíveis queixas decorrentes da deformidade dentofacial, secundárias à queixa principal, e que também acarretassem desconforto, 101 pacientes $(77,7 \%)$ relataram ter uma ou mais queixas secundárias que também contribuíram na decisão de procurar atendimento. No gênero feminino, as queixas secundárias foram, em ordem decrescente: dor orofacial $(37,3 \%)$, dificuldade mastigatória $(33,3 \%)$, estética $(18,7 \%)$, dificuldade fonética $(5,3 \%)$ e dificuldade de respiração nasal $(5,3 \%)$. No mas- 


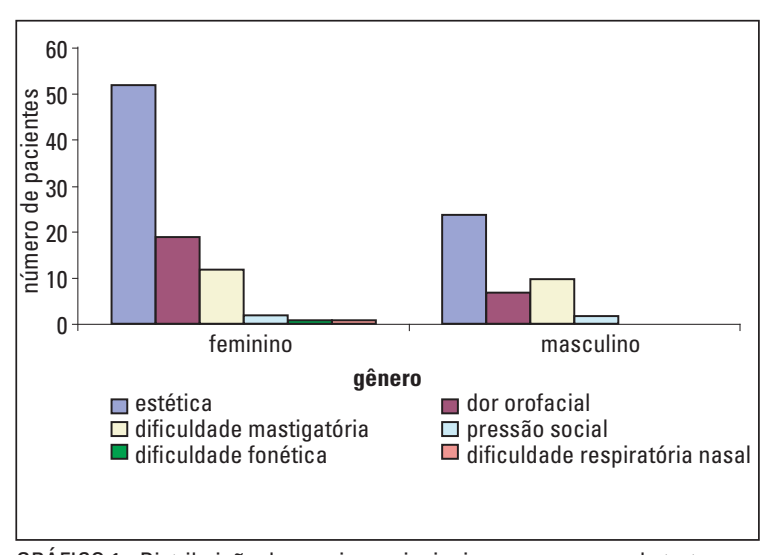

GRÁFICO 1 - Distribuição das queixas principais para a procura de tratamento conforme o gênero dos pacientes.

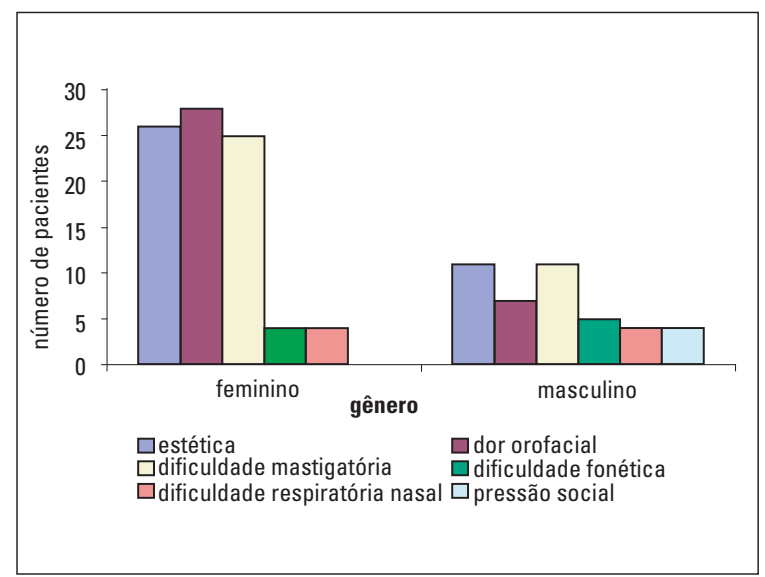

GRÁFICO 3 - Distribuição das queixas secundárias para a procura pelo tratamento de acordo com o gênero dos pacientes.

culino, essas queixas foram, em ordem decrescente: estética $(21,2 \%)$ e dificuldade mastigatória $(21,2 \%)$, dor orofacial $(13,5 \%)$, dificuldade fonética $(9,6 \%)$, dificuldade de respiração nasal $(7,7 \%)$ e pressão social (7,7\%) (Gráf. 3).

Em relação às queixas secundárias para a procura de tratamento de acordo com o tipo de deformidade dentofacial, os relatos de dor orofacial e dificuldade mastigatória foram predominantes no grupo 2 (35,1\% para ambas as queixas) e no grupo 1 (32,5\% e $30 \%$, respectivamente). No grupo 3 , houve predomínio de queixas de dificuldade mastigatória $(39,3 \%)$ e estética $(32,1 \%)$. Queixas funcionais de dificuldades fonética e respiratória

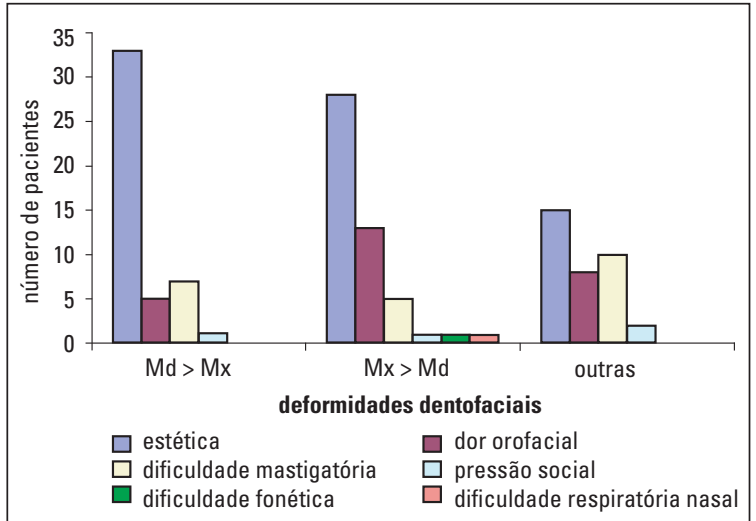

GRÁFICO 2 - Distribuição das queixas principais para a procura de tratamento de acordo com as deformidades dentofaciais.

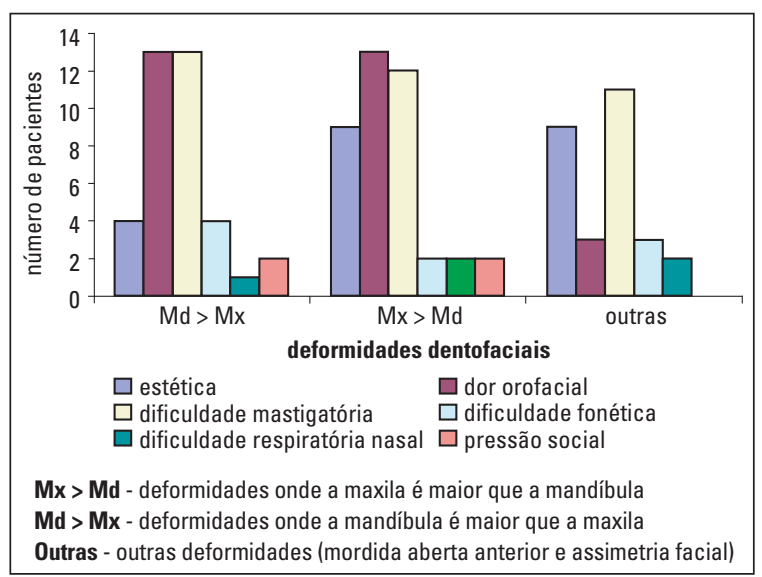

GRÁFICO 4 - Distribuição das queixas secundárias para a procura de tratamento de acordo com as deformidades dentofaciais.

nasal totalizaram $17,8 \%, 13,5 \%$ e $10 \%$, nos grupos 3, 2 e 1, respectivamente (Gráf. 4).

\section{DISCUSSÃO}

A casuística analisada apresentou a predominância do gênero feminino em relação ao masculino, em uma razão de 2:1, também observada por vários autores ${ }^{2,9,10,18}$. Outros autores apresentam casuísticas em que essa razão é ainda maior ${ }^{20,23}$, ou até mesmo menor ${ }^{25,27}$, mas sempre havendo a predominância do gênero feminino. Segundo Proffit, Phillips e Dann ${ }^{18}$, pacientes do gênero feminino se apresentam mais encorajadas e dispostas a procurarem e se submeterem ao tratamento ortodôntico 
e à cirurgia ortognática. Uma vez que o acometimento de deformidades dentofaciais não apresenta predominância de gênero, as pacientes do gênero feminino aparentam ter menor inibição em expressar seus anseios estéticos e em procurar alternativas de tratamento em favor dos mesmos ${ }^{9}$.

Com relação à idade dos pacientes na época que se submeteram à cirurgia ortognática, observamos a predominância da faixa etária de 20 a 39 anos para ambos os gêneros, similar a vários relatos ${ }^{5,16,20}$, mas contrastando com outros estudos em que as faixas etárias predominantes são menores ${ }^{3,7,11}$. Na casuística analisada, contribuíram, pela faixa etária observada à época da cirurgia, a conduta da equipe de cirurgiões da Instituição (CEDEFACE) em preferir operar pacientes que já terminaram a fase de crescimento ósseo e a opção do pesquisador em entrevistar pacientes maiores de 18 anos, excluindo da casuística os menores de idade.

Os relatos colhidos, em relação às queixas principais relatadas pelos pacientes, evidenciaram que os anseios de melhora estética foram predominantes em ambos os gêneros, resultando em 58,5\% para toda a casuística. Somando-se 20\% dos relatos encontrados entre as queixas secundárias, os anseios estéticos se fizeram presentes em $80 \%$ dos relatos, corroborando assim dados também observados na literatura ${ }^{8,9,24}$. Embora alguns autores relatem uma maior preocupação estética no gênero feminino ${ }^{7,10}$, os valores observados no gênero masculino se aproximam muito dos observados no feminino, sendo também os maiores daqueles relatados pela literatura citada.

As queixas de dor orofacial foram observadas em metade da casuística analisada, sendo a segunda queixa principal mais relatada, além de ser a queixa secundária mais citada pelo gênero feminino. A análise desses resultados indica um acometimento mais acentuado de dores orofaciais em pacientes do gênero feminino portadores de deformidades dentofaciais, estando de acordo com outros relatos $^{10,17,20}$. Magnusson, Ahlborg, Svartz ${ }^{15}$; Link e Nickerson $\mathrm{Jr}^{13}$ associam um maior número de relatos de dor orofacial na presença de retrognatismo mandibular, relação esta que foi observada na presente análise, encontrando-se uma distribuição relativamente maior do evento entre a categoria de deformidades $\mathrm{Mx}>\mathrm{Md}$, embora sem comprovação estatística.

A queixa funcional predominantemente relatada por ambos os gêneros foi a dificuldade mastigatória, tendo sido citada em 16,9\% das queixas principais e em $27,7 \%$ das queixas secundárias, observando-se um número proporcionalmente maior dessas queixas entre o gênero masculino, dados esses similares aos de Jacobson ${ }^{9}$; Kiyak et al. ${ }^{10}$, mas contrastando em muito com os observados por outros autores ${ }^{5,16,17}$, que afirmaram que as queixas de ordem funcional mastigatória sobrepujaram as de ordem estética, embora se observe nesses trabalhos casuísticas de maior faixa etária, onde se encontram maior número de pacientes apresentando dificuldades mastigatórias decorrentes da perda dos elementos dentários e osso alveolar de suporte.

A queixa funcional de dificuldade fonética foi citada entre as queixas principais uma única vez, por um paciente do gênero feminino portador de protrusão da maxila associada a severo retrognatismo mandibular, com impossibilidade de selamento labial. Assim como a queixa funcional de dificuldade respiratória nasal também foi citada uma única vez, por uma paciente portadora de excesso vertical da maxila. Entre as queixas secundárias, as dificuldades fonética e respiratória nasal também foram pouco citadas. Apesar de trabalhos como os de Turvey, Hall, Warren ${ }^{22}$; Erbe et al. ${ }^{6}$ identificarem, em pacientes portadores de hipoplasia de maxila e excesso vertical de maxila, altos índices de respiração bucal, os portadores dessas deformidades, curiosamente, pouco se queixaram da dificuldade de respiração nasal. Um fator que deve ser levado em consideração é a capacidade de adaptação funcional do sistema neuromuscular a novas situações, já que a deformidade dentofacial geralmente evolui de forma gradual, de acordo com o desenvolvimento esquelético, não acarretando, portanto, em difi- 
culdades funcionais tão evidentes aos pacientes. As porcentagens de queixas referentes a dificuldades fonéticas e respiratórias nasais se assemelharam às observadas por Jacobson ${ }^{9}$.

A respeito do pequeno número de queixas relacionadas à pressão social para realização da cirurgia ortognática, geralmente exercidas por familiares, tais pacientes merecem atenção especial, pois não visualizam os benefícios que seu tratamento pode lhes trazer. Segundo Barbosa et al. ${ }^{1}$, pacientes desmotivados ou apresentando pressões externas podem prestar pouca atenção ou até mesmo ignorar informações transmitidas durante o tratamento. Autores também afirmam que pacientes cujas motivações para a cirurgia ortognática não são evidentes tendem a apresentar maiores descontentamentos com os resultados obtidos ${ }^{9,20}$.

Analisando-se a distribuição das queixas principais e secundárias para a procura de tratamento, de acordo com os tipos de deformidade dentoesquelética, observamos uma homogeneidade entre as categorias, já que a maioria dos pacientes apresentou anseios tanto estéticos quanto funcionais, assim como no relato de Kiyak et al. ${ }^{10}$ Os anseios de melhora estética foram predominantes nas três categorias de deformidades, mais acentuadamente na categoria $\mathrm{Md}>\mathrm{Mx}$, provavelmente devido à característica de perfil côncavo gerada pelo aspecto protruído da mandíbula em relação à face do indivíduo.

Portanto, parece ser uma tendência atual a preocupação cada vez maior com os atributos físicos e estéticos, valores impostos pela sociedade atual em todos os segmentos, de acordo com os padrões culturais geográficos, os padrões temporais de moda e os conceitos de beleza vigentes.

\section{CONCLUSÃO}

- Os anseios de melhora estética foram os principais fatores para a procura pelo tratamento ortodôntico-cirúrgico em ambos os gêneros;

- A maioria dos pacientes apresentava anseios de melhoras funcionais paralelamente ao anseio estético;

- As queixas de dor orofacial apresentadas como motivo para a procura por tratamento ortodônticocirúrgico tiveram as maiores freqüências.

\title{
Assessment of aesthetic and functional complaints in patients with dentofacial deformities
}

\begin{abstract}
Aim: To assess the complaints referred by patients with dentofacial deformities as motivation for seeking orthodontic-surgical treatment. Methods: Were examinated 130 handbook of patients submited to surgical-orthodonthic treatment in CEDEFACE - Araraquara, with a post surgical period of 6 months and middle age of 30.9 years old. The deformities types' classification was done by telerradiography and photography analysis, subdivided in 3 groups according to its facial profiles carachteristics. The informations relative to complaints were obtained by questionnaire and subdivided in two categories: principal complaints and secundary complaints. To statistical analysis, central tendency measures were used and the variability measures to describe the numeric variables of age. The categoric variables of genders, dentofacial deformities and complaints were described by frequency distribution, with significance level of 5\%. Results: There was a predominance of females, in 2:1 proportion, and in the majority of the cases the deformities needed treatment only in the mandible or only in the maxilla. The convex profile was predominant in females (46\%) and the concave profile was most frequent in males (48.8\%). The principal complaints shows that the esthetical desire was predominant in males and females. Conclusion: The desire of esthetical improvement was predominant in both genders, and the majority of the sample showed additional desire to functional improvement. Orofacial pain was the second more related complaint, observed in half of the sample.
\end{abstract}

Key words: Dentofacial deformities. Orthognathic surgery. Treatment. Diagnosis. Complaints. 


\section{REFERÊNCIAS}

1 BARBOSA, A. L. B.; MARCANTONIO, E.; BARBOSA, C. E. M. GABRIELLI, M. F. R.; GABRIELLI, M. A. C. Psychological evaluation of patients scheduled for orthognathic surgery. J. Nihon Univ. Sch. Dent., Tokyo, v. 35, p. 1-9, 1993.

2. BAILEY, L. J.; HALTIWANGER, L. H.; BLAKEY, G. H.; PROFFIT, W. R. Who seeks surgical-orthodontic treatment: a current review. Int. J. Adult Orthod. Orthognath. Surg., Chicago, v. 16, p. 280-292, 2000.

3. CHENG, L. H. H.; ROLES, D.; TELFER, M. R. Orthognathic surgery: the patient's perspective. Br. J. Oral Maxillofac. Surg. Edinburgh, v. 36, p. 261-263, 1998

4. EGERMARK, I.; BLOMOVIS, T. J. E.; CROMV, I. K. U.; ISAKSSON, S. Temporomandibular dysfunction in patients treated with orthodontics in combination with orthognathic surgery. Eur. J. Orthod., Oxford, v. 22, p. 537-544, 2000

5. EK, E.; PERSSON, J.; LUNDGREN, S. Surgical correction of dentofacial anomalies: an evaluation of two patient groups with the aid of a questionnaire. Swed. Dent. J., Jönköping, v. 21, p. 101-110, 1997

6. ERBE, M.; LEHOTAY, M.; GÖDE, U.; WIGAND, M. E.; NEUKAM, F. W. Nasal airway changes after Le Fort I impaction and advancement: anatomical and functional findings. Int. J. Oral Maxillofac. Surg., Copenhagen, v. 30, p. 123-129, 2001.

7. FLANARY, C. M.; BARNWELL, G. M.; ALEXANDER, J. M Patient perception of orthognathic surgery. Am. J. Orthod., St Louis, v. 88, p. 137-145, 1985

8. HELDT, L. HAFFKE, E. A.; DAVIS, L. F. The psichological and social aspects of orthognathic treatment. Am. J. Orthod., St. Louis, v. 82, p. 318-328, 1982

9. JACOBSON, A. Psichological aspects of dentofacial esthetics and orthognaic surgery. Angle Orthod., Appleton, v. 54, p. $19-35,1984$

10. KIYAK, H. A.; HOHL, T.; SHERRICK, P.; WEST, R. A.; MCNEILL, R. W. BUCHER, F. Sex differences in motives for and outcomes of orthognathic surgery. J. Oral Surg., v. 34, p. 757-764, 1981

11. KIYAK, H. A.; McNEILL, R. W.; WEST, R. A. The emotional impact of orthognathic surgery and conventional Orthodonthics. Am. J. Orthod., St. Louis, v. 88, p. 224-234, 1985

12. LEE, A. S. Y.; WHITEHILL, T. L.; CIOCCA, V.; SAMMAN, N. Acoustic and perceptual analysis of the sibilant sound $s$ before and after orthognathic surgery. J. Oral Maxillofac. Surg., Philadelphia, v. 60, p. 364-372, 2002

13. LINK, J. J.; NICKERSON JR., J. W. Temporomandibular joint internal derangements in an orthognathic surgery population. Int. J. Adult Orthod. Orthognath. Surg., Chicago, v. 7, p. 161-169, 1992.

14. MAGNUSSON, T.; AHLBORG, G.; FINNE, K.; NETHANDER G.; SVARTZ, K. Changes in temporomandibular joint paindysfunction after surgical correction of dentofacial anomalies. Int. J. Oral Maxillofac. Surg., Copenhagen, v. 15, p. 707-714, 1986

15. MAGNUSSON, T.; AHLBORG, G.; SVARTZ, K. Function of the masticatory system in 20 patients with mandibular hypo- or hyperplasia after correction by a sagittal split osteotomy. Int. J. Oral Maxillofac. Surg., Copenhagen, v. 19, p. 289-293, 1990.

16. PANULA, K.; FINNE, K.; OIKARINEN, K. Incidence of complications and problems related to orthognathic surgery: a review of 655 patients. J. Oral Maxillofac. Surg., Philadelphia, v. 59 , p. 1128-1136, 2001

17. PHILLIPS, C.; BRODER, H. L.; BENNETT, M. E. Dentofacial disharmony: motivations for seeking treatment. Int. J. Adult Orthod. Orthognath. Surg., Chicago, v. 12, p. 7-12, 1997

18. PROFFIT, W. R.; PHILLIPS, C.; DANN, C. Who seeks surgicalorthodontic treatment? Int. J. Adult Orthod. Orthognath. Surg., Chicago, v. 5, p. 153-160, 1990

19. PRUITT, J. W.; MOENNING, J. E.; LAPP, T. H.; BUSSARD, D. A. Treatment of painful temporomandibular joint dysfunction with the sagittal split ramus osteotomy. J. Oral Maxillofac. Surg. Philadelphia, v. 60, p. 996-1002, 2002.
20. RIVERA, S. M.; HATCH, J. P.; DOLCE, C.; BAYS, R. A.; VAN SICKELS, J. E.; RUGH, J. D. Patient's own reasons and patientperceived recommendations for orthognathic surgery. Am. J. Orthod. Dentofacial Orthop., St. Louis, v. 118, p. 134-140, 2000

21. TUCKER, M. R. Correction of dentofacial deformities. In: PETERSON, L. J.; ELLIS III, E.; HUPP JR.; TUCKER, M. R. Contemporary oral and maxillofacial surgery. 2 nd ed. St. Louis: C. V. Mosby. 1993. cap. 25, p. 613-656.

22. TURVEY, T. A.; HALL, D. J.; WARREN, D. W. Alterations in nasal airway resistance following superior repositioning of the maxilla. Am. J. Orthod., St. Louis, v. 85, p. 109-114, 1994.

23. VALLINO, L. D. Speech, velopharyngeal function, and hearing before and after orthognathic surgery. J. Oral Maxillofac. Surg., Philadelphia, v. 48, p. 1274-1281, 1990

24. VAN STEENBERGER, E.; LITT, M. D.; NANDA, R. Presurgical satisfaction with facial appearance in orthognathic surgery patients. Am. J. Orthod. Dentofacial Orthop., St. Louis, v. 109, P. 653-659, 1996

25. WILMOT, J. J.; BARBER, H. D.; CHOU, D. G.; VIG, K. W. L. Associations between severity of dentofacial deformity and motivation for orthodontic-orthognathic surgery treatment. Angle Orthod., Appleton, v. 63, p. 283-288, 1993

26. ZARRINKELK, H. M.; THROCKMORTON, G. S.; ELLIS, E. III.; SINN, D. P. Functional and morphologic changes after combined maxillary intrusion and mandibular advancement surgery. J. Oral Maxillofac. Surg., Philadelphia, v. 54, no. 7, p. 828-837, July 1996.

27. ZHOU, Y.; HÄGG, U.; RABIE, B. M. Severity of dentofacial deformity, the motivations and the outcome of surgery in skeletal Class III patients. Chin. Med. J., Peking, v. 115, p. 1031-1034, 2002

\footnotetext{
Endereço para correspondência

Carla Maria Melleiro Gimenez

Rua Padre Duarte, 989 ap. 24 - Centro

CEP: 14801-310 - Araraquara / SP

E-mail: carlamg@yahoo.com
} 